\title{
Coronal oscillations in the vicinity of a sunspot as observed by GIS/CDS
}

\author{
C.-H. Lin ${ }^{1}$, D. Banerjee ${ }^{2}$, J. G. Doyle ${ }^{1}$, E. O'Shea ${ }^{1}$, and C. R. Foley ${ }^{3}$ \\ 1 Armagh Observatory, College Hill, Armagh BT61 9DG, N. Ireland \\ e-mail: chl@arm.ac.uk \\ 2 Indian Institute of Astrophysics, Koramangala, Bangalore 560034, India \\ 3 Mullard Space Science Laboratory, University College London, Holmbury St. Mary, Dorking, Surrey RH5 6NT, UK
}

Received 11 October 2004 / Accepted 5 January 2005

\begin{abstract}
In this paper, we present results from a time series analysis of data from the Grazing Incidence Spectrometer (GIS), onboard SoHO. Our observations were concentrated at the boundary between the quiet Sun and an active region, close to a sunspot plume. The dominant oscillations in all the lines studied are of periods longer than $5 \mathrm{~min}$. Although our observations were not taken directly above the sunspot, the 3-min oscillations, which are normally associated with the umbra, are seen in several spectral lines, suggesting some leakage perhaps via plume structures. The oscillations of coronal lines form wavepackets, and are intermittent with no obvious decay. The average time scale of the intermittence is of the order of 20 min, which would correspond to a source size of 2 arcsec.
\end{abstract}

Key words. Sun: corona - waves

\section{Introduction}

The oscillations in the atmosphere directly above various solar features have been extensively investigated by a range of instruments. Fludra $(1999,2001)$ investigated 3-min intensity oscillations above sunspots with the Solar and Heliospheric Observatory/Coronal Diagnostic Spectrometer (SoHO/CDS) by observing the chromospheric line $\mathrm{He}$ I and several transition region lines. He concluded that the 3-min umbral oscillations can occur both in the so called sunspot plumes (bright features seen in the transition region above a sunspot) and in the lower intensity plasma closely adjacent to the plumes. He found the spectral power to be contained in the $5.55-6.25 \mathrm{mHz}$ range. No oscillations were detected by him in the Mg IX $368 \AA$ line, suggesting that the 3-min oscillations do not propagate into the corona. Tziotziou et al. (2002) presented two-dimensional intensity and Doppler shift images computed at different wavelengths within the Ca II $8542 \AA$ line. Their power spectrum analysis shows a $6 \mathrm{mHz}$ frequency for the standing umbral oscillations only in the upper half part of the umbra. For the penumbra, they report a $3 \mathrm{mHz}$ frequency. They also conclude that the umbral oscillations are a localized phenomenon.

SoHO/SUMER (Solar Ultraviolet Measurements of Emitted Radiation) observations (in both intensity and velocity) have confirmed that the sunspot oscillations are prominent in transition region lines above the umbra (Maltby et al. 2001). They also state that the umbral oscillations are a localized phenomenon and that the 3-min oscillations fill the sunspot umbra in the transition region and tend to stop at the umbral rim. Support for the acoustic wave hypothesis was presented by Brynildsen et al. (1999a,b, 2004). They observed oscillations in intensity and velocity, and found the oscillations to be compatible with upwardly propagating waves.

More recently, O'Shea et al. (2002) and Brynildsen et al. (2002, 2004) have both presented joined observations of the 3 min umbral oscillations with TRACE (Transition Region and Coronal Explorer) and CDS. O'Shea et al. (2002) find oscillations at all temperatures from the temperature minimum, as observed by TRACE $1700 \AA$ up to the upper corona, as measured by the Fe XVI $335 \AA$ line with CDS. Both these studies report that the oscillation amplitude above the umbra increases with increasing temperature, reaches a maximum in the transition region and decreases for higher temperature lines. However, O'Shea et al. (2002) finds evidence for another increase in amplitude for lines formed above $1000000 \mathrm{~K}$. They interpreted the observations in terms of slow magneto-acoustic waves propagating upwards (based on time delays) along magnetic field lines. Brynildsen et al. (2004) have argued that their observed oscillations in the transition region can be modeled by upwardly propagating acoustic waves. Doyle et al. (2003) cautioned that in some instances, lines normally formed around $1000000 \mathrm{~K}$ can be shifted to significantly lower temperatures if a non-Maxwellian electron distribution is present. In a theoretical paper, Zhukov (2002) calculated the spectrum of eigenmodes of umbral oscillations. It was shown that the 3-min umbral oscillations are the p-modes modified by the magnetic field. 
Banerjee et al. (2002) detected both intensity and velocity oscillations in chromospheric and transition region lines as observed by CDS. They have shown that the 3-min intensity and velocity oscillations are a property of the umbra, and not just the sunspot plume. Banerjee et al. (2002) and Tziotziou et al. (2002) (observation by THEMIS (Thermal Emission Imaging System)) have also reported 5-min oscillations corresponding to the penumbra. Theories of active region oscillations can be found in the review by Bogdan (2000). From these studies one can conclude that the 3-min oscillations are confined to the region directly above an umbra while the 5-min oscillations are propagating waves outside of the umbra.

Acoustic waves propagating in coronal loops were first noticed in the high cadence EUV (Extreme UltraViolet) images of SoHO EIT (Extreme ultraviolet Imaging Telescope) observations (Berghmans \& Clette 1999). A number of propagating waves were also analyzed from TRACE data. The waves start in active regions, and propagate upwards into diverging, fanlike bundles of loops that fade out with height. However, no downward propagation was detected (De Moortel et al. 2000, 2002). Typical speeds of $v=122 \pm 43 \mathrm{~km} \mathrm{~s}^{-1}$ (De Moortel et al. 2002) were measured from TRACE $171 \AA$ data, where the mean sound speed is expected to be $c_{\mathrm{s}} \sim 147 \mathrm{~km} \mathrm{~s}^{-1}$ at $T \sim 1$ MK. Multi-wavelength observations from both EIT and TRACE confirm that the diverging fan structures consist of multiple loop threads with different temperatures and corresponding sound speeds (Robbrecht et al. 2001; King et al. 2003). While the waves that start further away from sunspots (in active region plages) have periods of $P=321 \pm 74 \mathrm{~s}$, the time periods of $P=172 \pm 32 \mathrm{~s}$ were found for loops rooted near sunspots, which coincide with the 3-min oscillations as discussed earlier (Maltby et al. 2001; Fludra 2001; Brynildsen et al. (1999a,b, 2004), The slow sound waves were also detected in optical wavelengths (in green lines at 5303 $\AA$ ) with spectroscopic methods using the Norikura white light coronagraph, with periods of $P=3-5$ mins and speeds of $v=100 \mathrm{~km} \mathrm{~s}^{-1}$ (Sakurai et al. 2002). In this paper, we present the analysis and results of an observation pointed at the boundary between the quiet Sun and an active region, corresponding to coronal loops fanning out of a sunspot.

\section{Observations and data reduction}

Our main data were obtained with the Grazing Incidence Spectrometer (GIS), which is one of the two components of CDS, on board SoHO. GIS consists of 4 detectors, with wave-

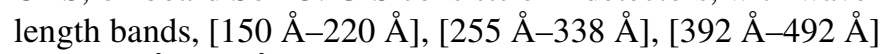
and [656 $\AA-784 \AA]$, respectively. The wide coverage of wavelength allows users of GIS data to investigate a complete range of temperatures. The other component of CDS is the Normal Incidence Spectrometer (NIS). A more detailed description of the GIS instrument can be found in Harrison et al. (1995) and the CDS Web-page.

The GIS sequence analyzed in this paper was taken as part of a Sunspot/Active region study on July 19, 2003. The observation was a 90-min sit-and-stare operation beginning at 04:03:08 UT. The target of the observation was AR405, which was a small active region located in the southern
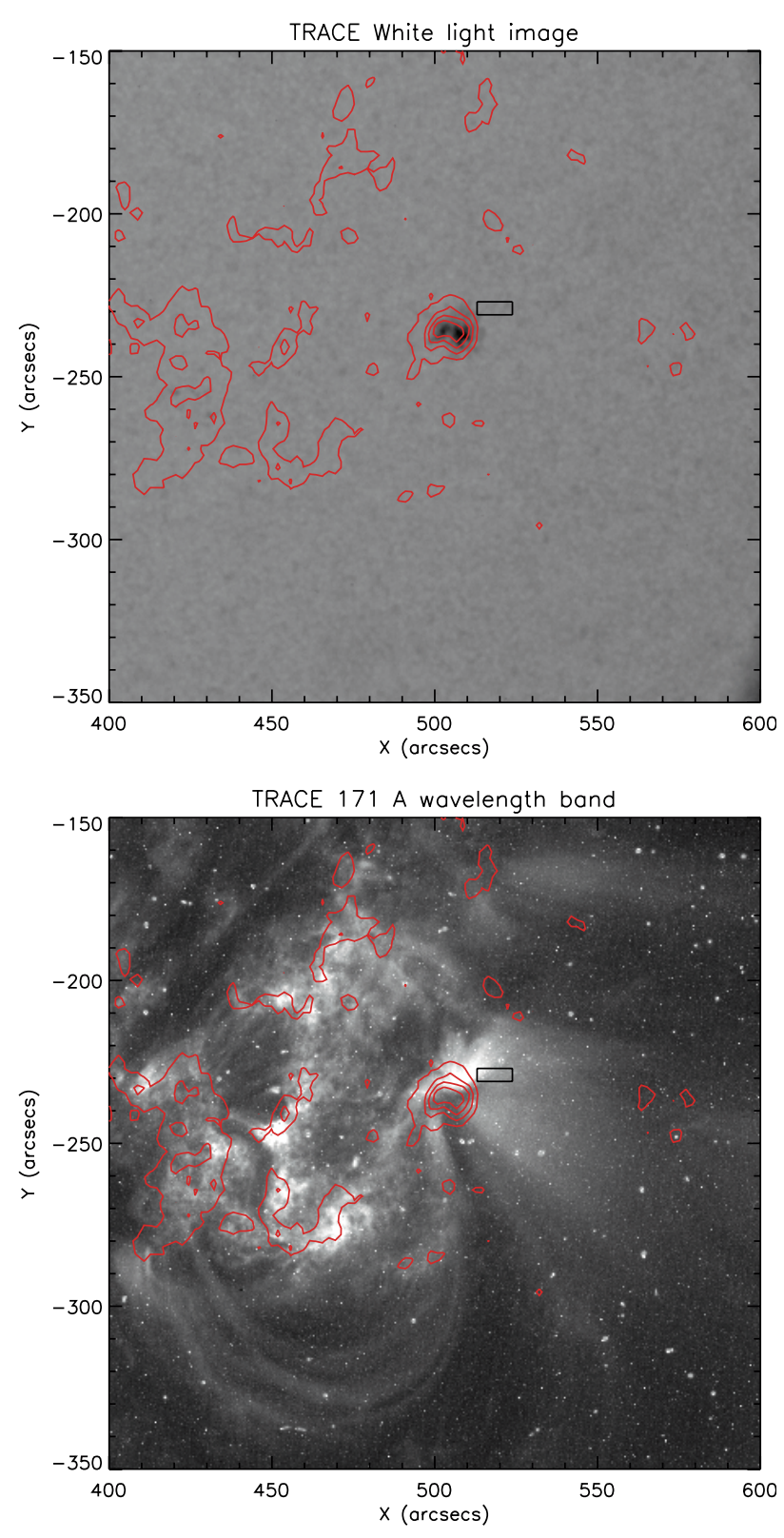

Fig. 1. An overview of the region of our observation. TRACE white light and TRACE $171 \AA$ channel $\left(\log T_{\mathrm{e}}=5.9\right)$ taken at 01:29 on 19 July 2003. The contours in the images represent the MDI magnetogram. The rectangular box represents our region of observations with GIS.

hemisphere. The solar rotation rate at this latitude implies a coverage of approximately 11 arcsec across the solar surface for the duration of the sequence. GIS measures the integrated light intensity of one pixel. In other words, the signal is a onedimensional time series without spatial resolution (astigmatic). To determine the location of the observation and to see the features we are analyzing, we incorporated the information from TRACE, NIS/CDS and MDI (Michelson Doppler Imager) images. However, there is typically a coordinate offset between images from different instruments. To correct for the offsets, we chose NIS coordinates as the standard coordinates, and shifted the images of other instruments until the features matched the features in the NIS image. 

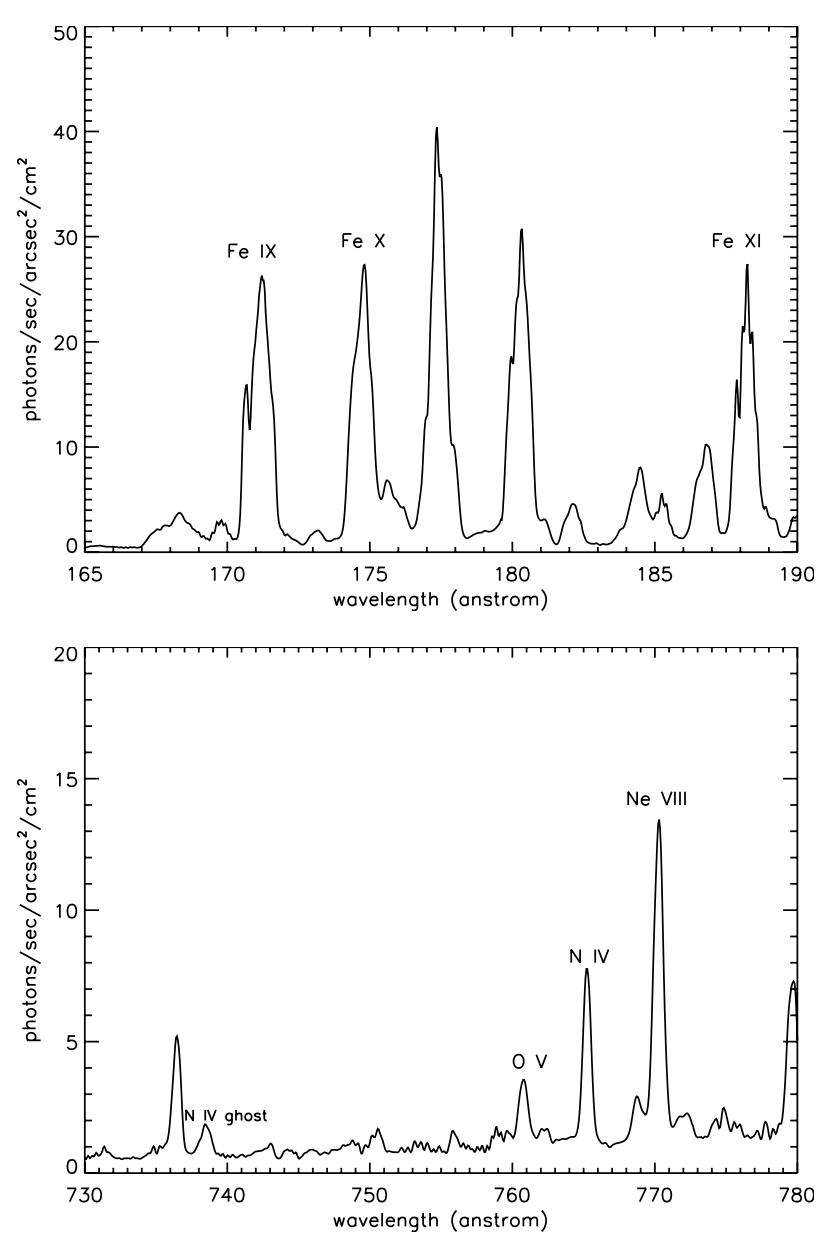

Fig. 2. Two examples of the GIS spectrum. The upper panel is from detector 1 and the lower panel from detector 4 .

Figure 1 shows a global view of the entire active region under discussion as seen with the TRACE white light and $171 \AA$ wavelength bands. The contours represent the distribution of magnetic fields as recorded by MDI. The small black rectangular box overlaid on these images at around location $513^{\prime \prime}<X<$ $524^{\prime \prime}, Y \approx-227^{\prime \prime}$ denotes our determined pointing location over the 90-min observation. The length of the vertical side of the box indicates the uncertainty in our determination. From a comparison of the magnetic contours and the position of the rectangular box, one can conclude that in the GIS field of view (FOV), no significant magnetic fields/structures are present at the photosphere level. We are, however, at the boundary between an active region and the "quiet Sun".

\subsection{Data processing}

NIS (taken between 03:26-03:36 and 05:37-05:47 UT, i.e. before and after the GIS sequence) and TRACE (taken at 01:29 UT, i.e. $2.5 \mathrm{~h}$ before the GIS sequence) images were calibrated by respective standard calibration routines (see O'Shea et al. 2002 for further details). The offset between NIS1 and NIS2 was corrected for by the routine nis_rotate. No calibration was applied to MDI images. GIS data were calibrated and corrected for instrumental effects using routines from the Solar Software library. The data were first converted from FITS format to a Quick Look Data Structure (QLDS) by readcdsfits, and the wavelengths were calibrated through restore_wavecal and pix2wave. Next, the two main instrumental errors to be corrected are "fixed patterning" and "ghosting". The effect of fixed patterning, caused by electronic read-out errors, is reduced by smoothing the line profiles by the procedure gis_smooth. "Ghosting" is when the intensities of certain lines are partially shifted and recorded at incorrect locations on the detector, leading to incorrect line intensities (CDS software note No. 55 and Del Zanna 1999). The ghost_buster routine can automatically correct approximately half of the ghosting lines. The remaining ghosts can be further corrected through a manual correction mode. The routine can also output the regions that are free of any ghosting effects. Finally, the gis_calib procedure corrects the electronic effects on line intensities. The unit of the intensity is converted into photons/s/arcsec $2 / \mathrm{cm}^{2}$ (see CDS Software note No. 55 for the details of the errors and the calibration of GIS data)

Figure 2 shows the averaged spectra from detector 1 (upper panel) and 4 (lower panel). The lower panel in the figure contains an example of GIS ghosting. The line at $738 \AA$ is a ghost from the N IV $765 \AA$ line, that is, part of $765 \AA$ photons were allocated to $738 \AA$, where no line is expected to exist.

The fitting of the spectral lines was repeated by three different schemes:

- gis_fit: this is an automatic GIS fitting routine which fits a fixed set of lines.

- Averaged spectrum fit: in this scheme, we first fitted the averaged spectrum (i.e., the average of the 90 spectra) by xcfit to obtain fitting parameters. These averaged parameters were then applied to all 90 spectra by using xcfit_block.

- Representative spectrum fit: this scheme essentially follows the same procedure as in averaged spectrum fit, except that, instead of an averaged spectrum, a selected representative of the 90 spectra is used. We used two representative spectra to produce two independent sets of fitting results.

Lastly, the fitted lines that show large fluctuations in line widths and/or positions in any of the three fitting schemes were discarded. To obtain the correct intensity for the ghosting line $765 \AA$, we first fitted $738 \AA$ and $765 \AA$ separately, and then added the line intensities of the two (Del Zanna 1999). The lines selected for further analysis are listed in Table 1. The temperature of ionization equilibrium of each line was calculated from CHIANTI synthetic spectra, ch_ss, using mazzotta_etal_ext.ioneq as the ionization fractions file and active_region.dem as the differential emission measure file. After the fitting, the total intensity of each selected spectral line was then computed.

\subsection{Wavelet analysis}

Wavelet transform is analogous to localized Fourier transform. It provides information on the temporal dependence of a signal. Because the coronal oscillations often vary over time, the 

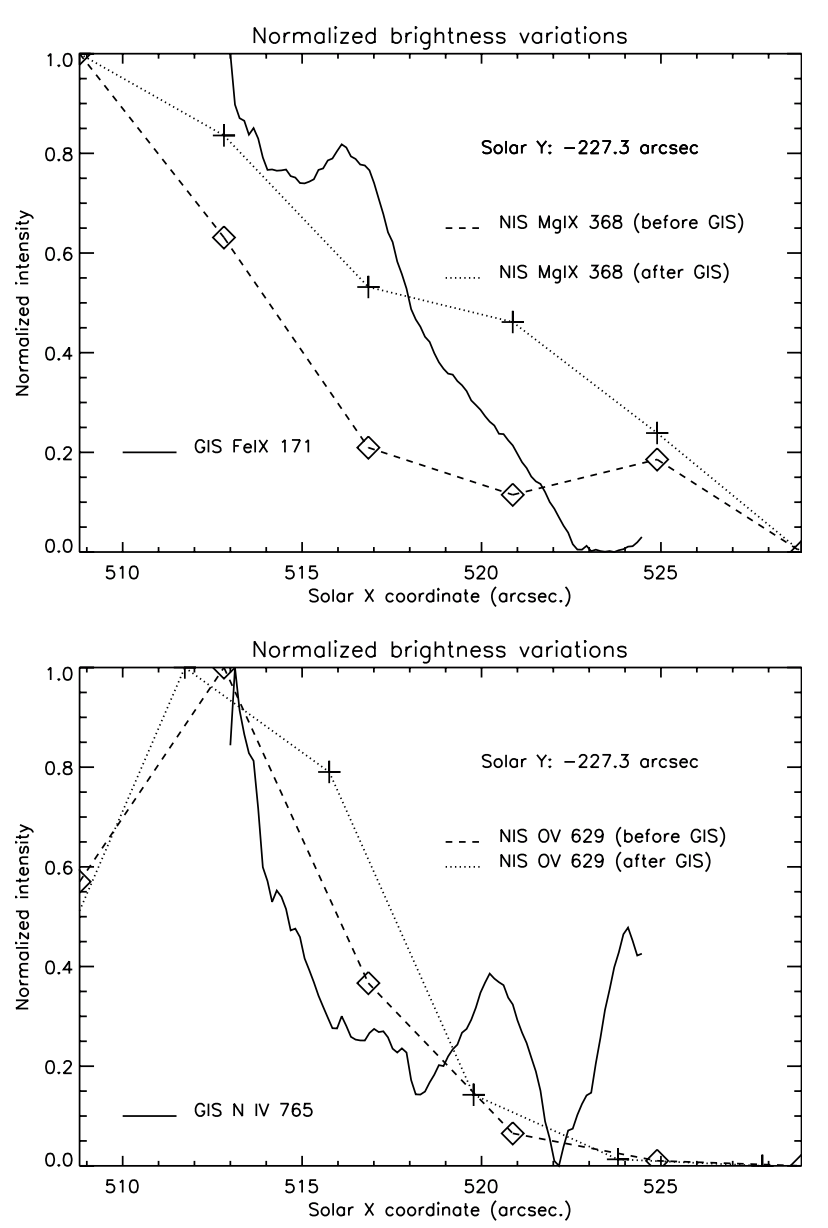

Fig. 3. The normalized trend of the intensity variation over time of GIS Fe IX $171 \AA$ and the brightness variations in NIS Mg IX $368 \AA$ line for a region 513" $<X<525^{\prime \prime}, Y \approx-227^{\prime \prime}$ (top panel) and GIS N IV $765 \AA$ and NIS O V $629 \AA$ (bottom panel). Note that the time sequence of the GIS light curve has been inverted because the GIS observation scanned the solar surface from west to east.

wavelet transform, which allows analysis on localized variations, is more suitable than the traditional Fourier analysis for our study. Details on the wavelet analysis may be found in Torrence \& Compo (1998) and O'Shea et al. (2001). To determine whether or not the oscillations found in the analysis are real, we implemented a randomization method (Nemec \& Nemec 1985) to estimate the significance level of the peaks in the wavelet spectrum. The significance level resulting from the randomization method has been compared with the significance level output from the procedure wavelet. The comparison shows that the former method is more strict than the latter. Nevertheless, the two results are consistent.

\section{Results and discussion}

\subsection{The region of observation}

By comparing the two NIS Mg IX $368 \AA$ images taken before and after the GIS observation, we found that the brightness variation profile of this line at the vicinity of AR405 had significantly changed over the time of the observation. This indicated that the region was very dynamic at these temperatures.
Table 1. The GIS fitted lines.

\begin{tabular}{lcc}
\hline \hline Ion & Wavelength & $\log T_{\mathrm{e}}$ \\
\hline Fe XV & 284 & 6.3 \\
Fe XI & 188 & 6.1 \\
Fe X & 174 & 6.0 \\
Fe IX & 171 & 5.9 \\
Ne VIII & 770 & 5.9 \\
Ne VIII & 780 & 5.9 \\
Ca IX & 466 & 5.8 \\
Ne VII & 465 & 5.8 \\
O V & 760 & 5.4 \\
N IV & 765 & 5.1 \\
He II & 304 & 4.9 \\
\hline
\end{tabular}

Therefore, the TRACE 171 image, which was taken $2.5 \mathrm{~h}$ earlier than the CDS data, does not represent the conditions at the time of the GIS observation. Consequently, instead of using TRACE 171, we compared the GIS N IV $765 \AA$ light curve with the NIS O v 629 A intensity variations. After examining the variations in the region $-214^{\prime \prime}<Y<-229^{\prime \prime}$, we present the best match in Fig. 3. In the figure, the normalized trend of the intensity variation (cf. Sect. 3.2 for the computation of the trend) of GIS Fe IX $171 \AA$ is compared with the normalized intensity variation of NIS Mg IX $368 \AA$ line in the region $513^{\prime \prime}<X<525^{\prime \prime}$ and $Y \approx-227^{\prime \prime}$, using the NIS rasters taken before and after the GIS sequence. Also, we make the same comparison using GIS N IV $765 \AA$ and NIS O V $629 \AA$ A. Note that the sit-and-stare GIS observation scanned the region from west to east (i.e., from greater to smaller solar $X$ coordinates); hence, the GIS curve in the figure is inverted in order to be consistent with the solar $X$ coordinate in the $x$ axis. The close resemblance of the nature of the variations suggests that we have corrected the offsets between different instruments and have accurately determined the pointing of our GIS observation. This is in close agreement with the latest GIS pointing information given by the GIS team.

In Fig. 4, we show an enlarged view of our region of observations as seen by different instruments. The contours represent the distribution of magnetic field as recorded by MDI, and the small rectangular box marks the pointing location of GIS during the 90-min observation. The arrows (in the right most panel) indicate the locations at which a completely new $4^{\prime \prime} \times 4^{\prime \prime}$ area enters the field of view of the GIS slit. The MDI magnetogram suggests that the region is smooth and, apart from the sunspot, lacks features at the photospheric level. The TRACE $171 \AA$ image shows a congregation of footpoints (presumably the end points of coronal loops). The loops seem to be radiating out $\left(X \approx 510^{\prime \prime}\right.$ and $Y \approx-235^{\prime \prime}$ ) (northward and southward) from the sunspot umbra. From the NIS O v $629 \AA$ image (third panel from the left in Fig. 4), we can see a region of strong emission (the brightening) which corresponds to a sunspot plume. Interestingly, this region looks dark in the corona (compare with the first panel in Fig. 4). The velocity map of NIS O v 629 (the right most panel of Fig. 4) shows two pairs of red and blue shifted material surrounding the brightening. The Doppler velocity computed from the blue/red shifts 

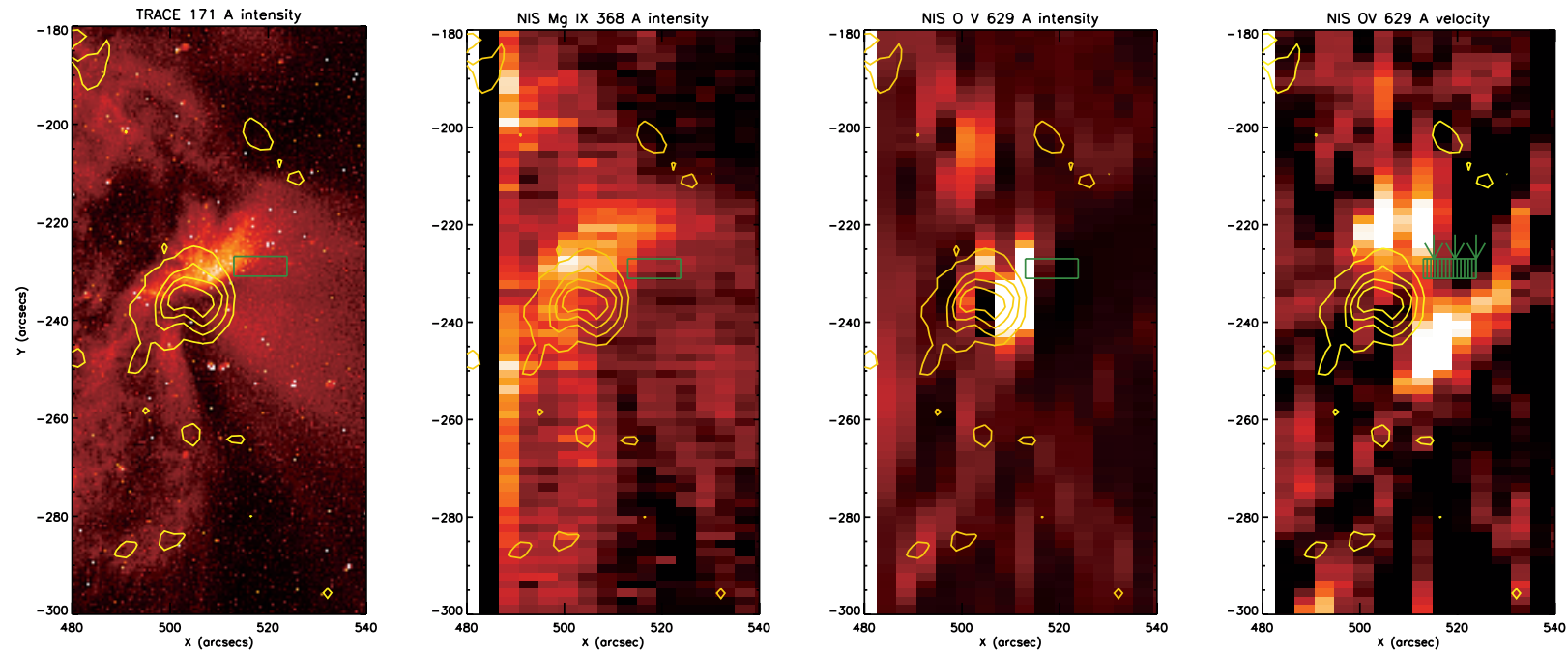

Fig. 4. An enlarged view of the region of observation from different instruments, as denoted on the top of each panel. The NIS image used is that taken after the GIS sequence. The contours represent magnetic field concentrations as revealed by a MDI magnetogram. The rectangular box indicates the FOV of the entire GIS observation. In the velocity map, greater red shifts are represented by brighter colors. The lines mark the time steps, and the arrows above the rectangular box indicate the locations at which a completely new $4^{\prime \prime} \times 4^{\prime \prime}$ area enters the field of view of the GIS slit.

Table 2. The main oscillation periods and the global probability levels in the three passbands of our selected lines.

\begin{tabular}{cccccccc}
\hline \hline $\begin{array}{c}\text { Line } \\
(\AA)\end{array}$ & $\begin{array}{c}\text { Temp. } \\
\left(\log T_{\mathrm{e}}\right)\end{array}$ & $\begin{array}{c}\text { Period } \\
(\mathrm{s})\end{array}$ & $\begin{array}{c}\text { Global prob. } \\
(\%)\end{array}$ & $\begin{array}{c}\text { Period } \\
(\mathrm{s})\end{array}$ & $\begin{array}{c}\text { Global prob. } \\
(\%)\end{array}$ & $\begin{array}{c}\text { Period } \\
(\mathrm{s})\end{array}$ & $\begin{array}{c}\text { G00 s } \\
\text { Global prob. } \\
(\%)\end{array}$ \\
\hline Fe XV 284 & 6.3 & 181 & 96.5 & 332 & $99-100$ & 1218 & $99-100$ \\
Fe XI 188 & 6.1 & 166 & 92.5 & 394 & 99.5 & 664 & $99-100$ \\
Fe X 174 & 6.0 & 181 & 83.5 & 430 & 98 & 1116 & $99-100$ \\
Fe IX 171 & 5.9 & 166 & 96.5 & 362 & $99-100$ & 1218 & $99-100$ \\
Ne VIII 770 & 5.9 & 181 & 97 & 332 & $99-100$ & 789 & $99-100$ \\
N IV 765 & 5.1 & 166 & 78.0 & 469 & $99-100$ & 1024 & $99-100$ \\
\hline
\end{tabular}

is approximately $40 \mathrm{~km} \mathrm{~s}^{-1}$. Our comparison of the GIS light curve with the brightness variations of other images (cf. Fig. 3) indicates that the boundary of the upper red and blue shifts is the region of our GIS observation, as marked by the rectangular box. After a closer inspection of the velocity map and the other images, we suggest that our GIS observation was obtained at the crossing of loops with opposite flow directions. Essentially our FOV crosses a system of coronal loops connected to the neighbouring sunspot.

\subsection{The oscillations}

To ensure that our analysis and results of the oscillations are unambiguous, we discarded the weaker lines; the lines that might be blended and/or contaminated by near-by lines; the lines that are close to the boundary of detector windows; and the lines that have ambiguous ionization temperatures. The lines that passed our selection are listed in Table 2 . The intensity variations over time (light curves) of several selected lines are plotted in Fig. 5 (note that these curves are also over a spatial region of $\approx 11^{\prime \prime}$ and are thus not located at a single location). The thick dashed line in each panel represents the trend of the intensity variation, computed by a 15 -pt running average.
The oscillations were extracted by subtracting the trend from the intensity (de-trending). The errors in the intensity amplitudes are computed based on CDS Software Note No. 49.

In some data, we found large impulses which stood out, such as that occurred in the first 5 min of the Fe XV 284 signal (Fig. 5). We filtered these out using a preliminary filtering procedure, to prevent these affecting the significance of the real oscillations.

Figure 6 illustrates four examples of the wavelet transform on the unfiltered oscillations. In each example, the top panel shows the original intensity variations (after subtracting the trend) in counts/s/area along with the errors; the lower left panel is the wavelet spectrum; and the lower right panel is the global wavelet spectrum, which is the sum of the wavelet power over time at each oscillation period. In the wavelet spectrum, the dark-colored regions show the locations of the highest power. Only locations that have a probability greater than 95\% are regarded as being real, i.e., not due to noise. Crosshatched regions indicate the "cone of influence" (COI), where edge effects become important (see Torrence \& Compo 1998). The dashed horizontal lines in the global wavelet spectrum marks the higher period cut-off (1991 s) due to edge effects. The multiple peaks in the global wavelet spectra indicate that 

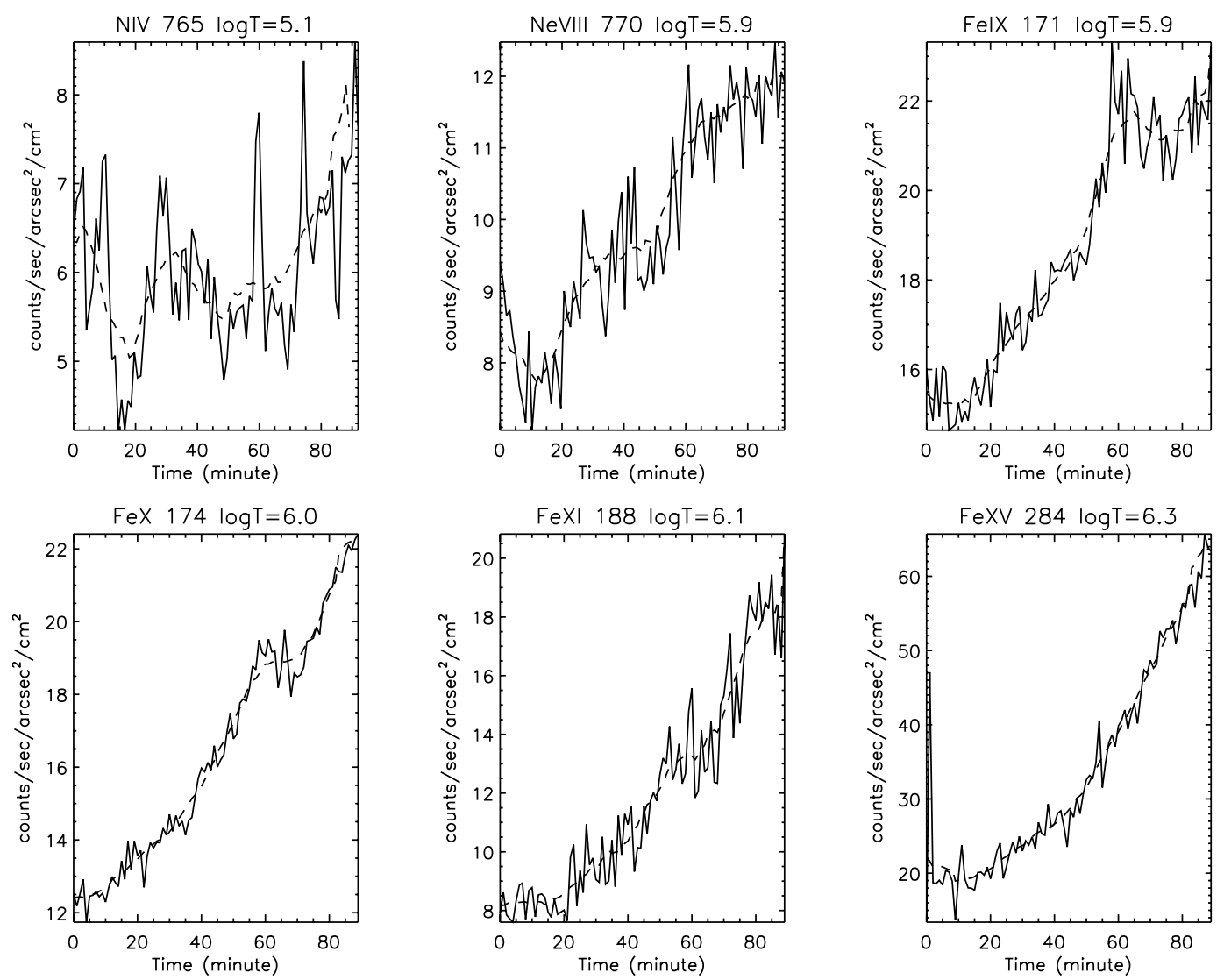

Fig. 5. The light curves (i.e., the intensity variations over time) of different temperature lines. The dashed line in each plot represents the trend. Note that, because of the sit-and-stare operation, the GIS observation scanned the solar surface from west to east.

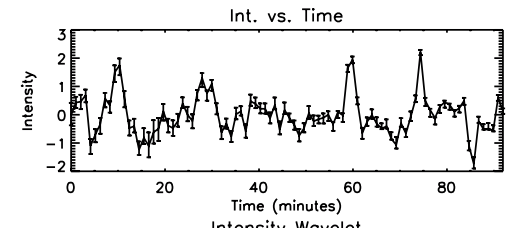

$N$ IV $765 \log T=5.1$

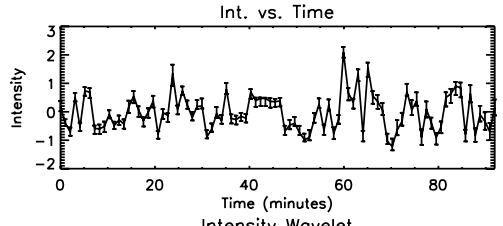

FelX $171 \log T=5.9$
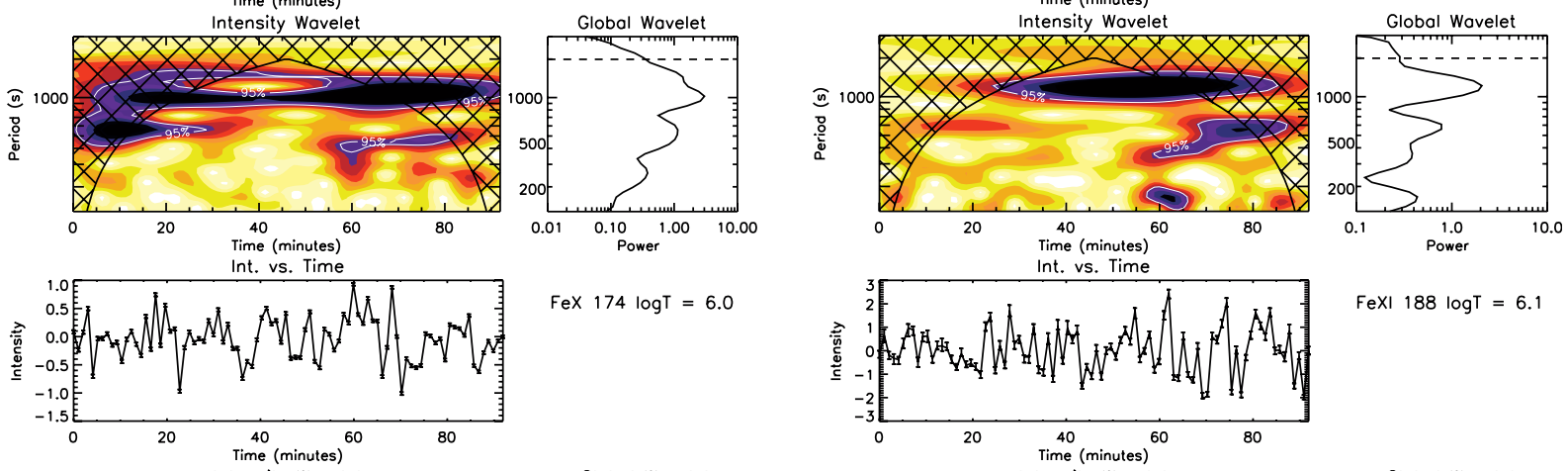

FeX $174 \log T=6.0$

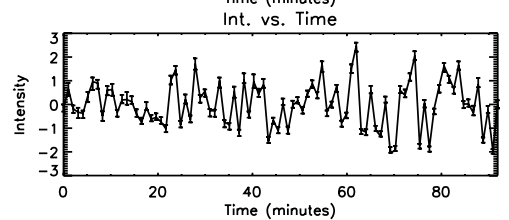

FeXI $188 \log T=6.1$
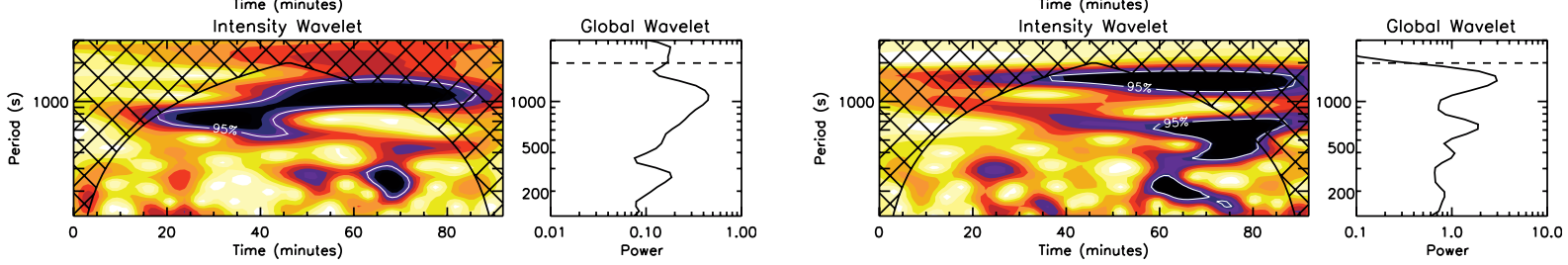

Fig. 6. Examples of the wavelet analysis on intensity variations recorded by different temperature lines, as denoted in the upper right conner of each example. In each example, the upper left panel shows the de-trended intensity variation of the line (cf. Sect. 3.2 for the de-trending process) with associated error bars; the lower left panel is the corresponding wavelet transform; and the lower right panel represents the wavelet power integrated over time. The unit of intensity is counts/sec/area. In the wavelet transform panels, white contours circle the region above the 95\% significance level, and the black cross-hatched area indicates the cone of influence, i.e., the region affected by edge effects. 

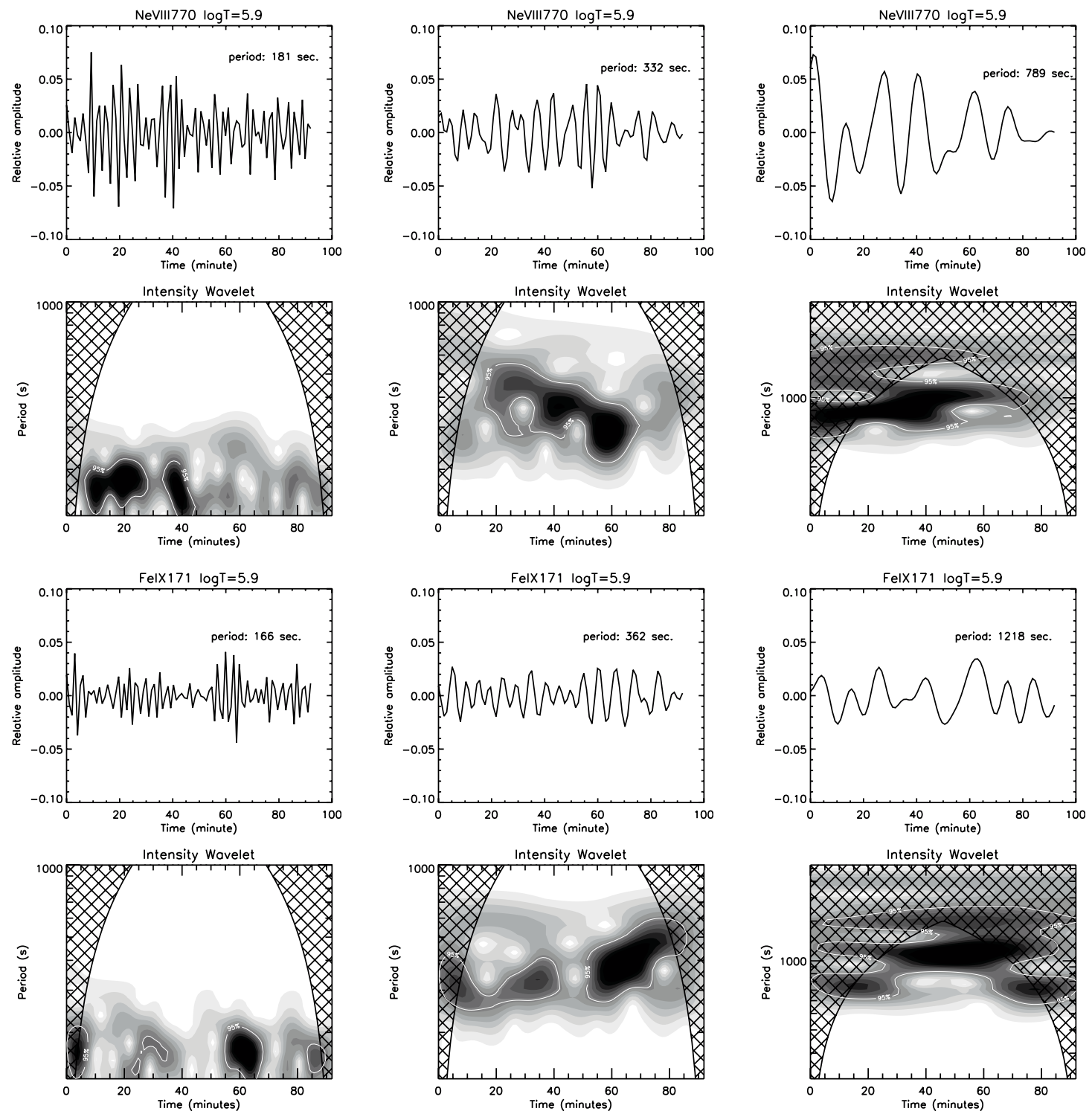

Fig. 7. Filtered oscillations (first and third rows) and the corresponding wavelet spectra (second and forth rows) of Ne VIII $770 \AA$ and Fe IX $171 \AA$ A. The passband of each column, from left to right, is [0-250] s, [250-500] s, and [500-2000] s. The cross-hatched regions on the two sides of each wavelet spectrum mark the "cone of influence" (COI). The white contours in the spectra circle the regions with $95 \%$ significance level.

the signals are often composed of oscillations of different periods. Examining the global wavelet spectra, we see that the wavelet powers of all the selected lines are concentrated in the long-period band. However, we found that the power of global wavelet spectra of relative oscillations (i.e., oscillations/trend) is more evenly distributed among different periods. The only exceptions are $\mathrm{N}$ IV and Fe X, for which the power is still concentrated in the long-period band. The reduction of the power in the long-period band indicates that such long-period oscillations are partially a result of the variation in the line intensity.

To examine the features and properties of the oscillation of a specific period, it is thus necessary to apply a bandpass filter to the signal. The length of our signal and the principle of wavelet transform impose an upper cut-off oscillation period of $1991 \mathrm{~s}$. Hence, we considered only the oscillations with periods in the range 0-2000 s. We implemented three passbands: [0-250] s, [250-500] s, and [500-2000] s, which are to extract 3-min, $5-\mathrm{min}$ and $10-15 \mathrm{~min}$ (i.e., $5 \mathrm{mHz}, 3 \mathrm{mHz}$ and $1 \mathrm{mHz}$ ) oscillations, respectively. Doyle et al. (1998) showed that the effect of sit-and-stare operation is to spread the power of each oscillation frequency. At the location of our observation, the rotation rate, 4 arcsec per $36 \mathrm{~min}$, results in a frequency spread $\approx 0.46 \mathrm{mHz}$. Therefore, our passbands are sufficiently spaced such that there is no cross talk between the oscillations in different bands.

The filtered relative oscillations and wavelet spectra for two representative cases corresponding to the Ne VIII and Fe IX are plotted in Fig. 7. The filtering indeed enhances the visibility of the weaker 3-min oscillations. 
The main oscillation period (i.e., the period with highest global wavelet power) and its global probability level in each passband of the selected lines are listed in Table 2. Table 2 shows that oscillations with a period of $5 \mathrm{~min}$ or longer are more significant than the 3-min oscillations in all the lines. Nevertheless, 3-min oscillations are still reasonably prominent (i.e. probability level $>90 \%$ ) in most lines, except for N IV 765 and Fe X 174.

There are several characteristics in the obtained oscillations common in all lines:

1. Although our observation was not directly above the sunspot, the 3-min oscillations, which have heretofore been considered to exist only in the umbra, are seen in many of our selected lines except for the aforementioned N IV and Fe XI lines. These 3-min oscillations in our results are often isolated oscillations, with a duration less than $\approx 20 \mathrm{~min}$. This could equally imply that the oscillations are confined to regions of around $2^{\prime \prime}$ in size.

2. The oscillations are intermittent and wavepacket-like with no obvious decay. Such intermittency, although appearing in all lines, is especially prominent in the Fe IX 171 and Ne VIII 770 lines. The average time scale of the intermittence is of the order of $20 \mathrm{~min}$, again perhaps implying a source size of around $2^{\prime \prime}$.

3. In the [500-2000] s passband, we found clean and prominent 10-15 min oscillations in all the lines we examined. These oscillations often last longer than 40 min without decay although their periods and amplitudes vary over the time (cf. Ne VIII). The uniformly high level of significance of these oscillations (cf. Table 2) is a result of their long duration. In addition, an oscillation with a period $\approx 20 \mathrm{~min}$ $(\approx 1200 \mathrm{~s})$ is visible in many lines. Because of the width of GIS slit and the sit-and-stare operation, a point source enters and exits the FOV of the slit in $36 \mathrm{~min}$. Therefore, a prominent oscillation that has a period $\approx 20 \mathrm{~min}$ is likely due to periodic spatial variations. This is consistent with our suggestion in the first two points, that is, the intermittence of both 3-min and 5-min oscillations is $\approx 20 \mathrm{~min}$ indicating a source size of $2^{\prime \prime}$. However, we do not rule out the possibility that the observation was crossing a uniform oscillation source that is larger than the size of the detector slit.

The GIS detectors detect the integrated light intensity over a $4^{\prime \prime} \times 4^{\prime \prime}$ area. As the Sun rotates, new areas gradually enter the field of view of the slit. Due to the solar rotation rate at the latitude of our observation, the slit covers a completely new $4^{\prime \prime} \times 4^{\prime \prime}$ region every $36 \mathrm{~min}$. In Fig. 4, the arrows in the velocity map indicate these locations. Based on the determined pointing, the slit approaches the end of the blue shift region at $\sim 24$ th min, and is crossing the boundary of blue/red shift from the 36th to 48th min. In the last $36 \mathrm{~min}$, the boundary of the plume is in the FOV of the slit. We believe we have a scenario in which the detector crosses loops with opposite flows and eventually enters a sunspot plume. In such a scenario what could happen to the wavelet power spectra? While most of the lines we analyzed do not exhibit prominent features at these locations, some lines do show a change. For example, Ne VIII 770 shows significant (i.e. $>95 \%$ probability level) 3 -min oscillations only in the first $40 \mathrm{~min}$, that is, before the slit fully enters the red shift region. Although an isolated packet of oscillations occurs around $\approx 60$ th $\min$ (when the boundary of the plume enters the FOV) in some lines (e.g., Fe IX 171), the oscillations are not significantly stronger than oscillations at other locations.

\subsection{Summary}

The oscillations with periods of $5 \mathrm{~min}$ and longer are the dominant oscillation in all of our selected lines. Although our observation was not directly above the umbra, the 3-min oscillations are present (i.e. probability level higher than 95\%) in many lines that we studied. These 3-min oscillations appear as isolated wavepackets with durations less than $\approx 20 \mathrm{~min}$, corresponding to a source of $\approx 2^{\prime \prime}$. In contrast, the wavepackets of dominant long-period oscillations often last longer than $30 \mathrm{~min}$.

Although the edge of the plume was in the FOV during the last $36 \mathrm{~min}$ of our observation, there is no significant enhancement in the oscillation amplitudes towards the end of our signal. We found that the locations where a wavepacket starts and stops do not show apparent correlation with temperature variation, and are not always associated with the locations of the red/blue-shift boundary and the red/blue-shift zones. The lack of correlation between the oscillations and temperature variations could indicate that the loops are inclined or twisted or that the thermal structures in the loops are not stratified. Since the Doppler velocity computed from the blue/red shifts indicates that the shifts are likely due to flows in the loops, the indifference of the shifts to the oscillations suggest that our oscillations are not significantly affected by such flows.

\section{Conclusions}

The detection of oscillations in coronal loops is critical in determining the presence of waves in the corona and their relevance to the problem of coronal heating. Using the UltraViolet Coronagraph Spectrometer UVCS/SoHO instrument, signatures of compressional waves in plumes were first detected high above the limb by Ofman et al. (1997) and subsequently by DeForest \& Gurman (1998). De Moortel et al. (2000) reported on the detection of similar propagating oscillations observed in coronal loops in the TRACE $171 \AA$ passband, which they suggest to be propagating slow magnetoacoustic waves. The results of De Moortel et al. (2002) suggest that not only the 3-min umbral oscillations but also the global 5-min oscillations are present in the corona. An extensive review of a totally different type of oscillatory phenomenon, flare-excited transverse oscillations of coronal loops, was presented by Nakariakov et al. (1999), Schrijver et al. (2002) and Aschwanden et al. (2002). Most of the longitudinal oscillations as recorded by TRACE so far have been found in large quiescent coronal loops that emanate from the edge of active regions. This makes our results from GIS very much complementary to what De Moortel et al. have reported so far. They also pointed out that no oscillations were found in the actual active region loops but whether these shorter, more active loops do not support such oscillatory signals or whether the signals are just obscured by some other event remains unclear. 
In our present observation, we have a complicated topology, but it appears that these loops are fanning out of the sunspot. So far TRACE have reported oscillations only at the footpoints of these coronal loops (De Moortel et al. 2002; Brynildsen et al. 2004) and in this study, for the first time, we are reporting oscillations from these loops away from the footpoint, suggesting they are caused by propagating waves. De Moortel et al. (2002) pointed out that loops that are situated above sunspot regions display intensity oscillations with periods of the order of $172 \pm 32 \mathrm{~s}$, whereas oscillations in nonsunspot loops show periods of the order of $321 \pm 74 \mathrm{~s}$. In our observation we find both the 3-min and the 5-min periodicity. Previous work has suggested that the 3-min oscillations are confined to sunspot plumes. Sunspot plumes are defined (Noyes et al. 1985) as regions over-lying a sunspot and having a factor of 5 to 10 increase in the intensity of transition lines over the surrounding region. The brightest region visible in the NIS O v $629 \AA$ image is a factor of 6 brighter than the location observed by the GIS slit, which is a factor of $\approx 2.5$ greater than the quiet Sun. Thus, the region observed in the present GIS observations would not meet the normally accepted definition of a plume region. Nevertheless, TRACE 171 images show coronal loops extending from the sunspot umbra towards our GIS location. Furthermore, the NIS O V image shows that the bright structure (the plume) extends out to the FOV of the GIS observation; thus, some leakage of wave activity must be taking place from the sunspot plume. This is consistent with the findings of Fludra (2001) who found that the 3-min oscillations also occur in the low-intensity plasma adjacent to plumes.

The long period $(P=10-15 \mathrm{~min})$ slow waves have been observed so far only in open magnetic structures in coronal holes (DeForest \& Gurman 1998; Ofman et al. 1997; Banerjee et al. 2001). In this work, we find the presence of long periodicity in the vicinity of a sunspot within coronal loops, though we are not sure about the magnetic topology. The propagating intensity disturbances in coronal loops as seen by TRACE has been interpreted as being due to acoustic waves (Klimchuk et al. 2004). They simulate the propagation of the waves in a 1-D hydro code and conclude that the periodic nature and the speed of these disturbances suggest that they are traveling acoustic waves. We should remind the readers that, in a low beta plasma, the pure acoustic waves and the slow magnetoacoustic waves can behave very similarly. Thus, taking into account all the observed properties, we can interpret our observed oscillations as propagating slow magneto-acoustic waves or pure acoustic wave. Furthermore, our observation indicates that the drivers are probably near the footpoints and definitely not flare driven.

Acknowledgements. We would like to thank the CDS and TRACE teams at Goddard Space Flight Center for their help in obtaining the present data. CDS and MDI are part of SoHO, the Solar and Heliospheric Observatory, which is a mission of international cooperation between ESA and NASA. D.B. wishes to thank the Royal Society London and the DST, India. His visit to the Armagh Observatory was supported within the India-UK Science Networks program. Research at Armagh Observatory is grant-aided by the N. Ireland Dept. of Culture, Arts and Leisure. This work was supported by a PRTLI research grant for Grid-enabled Computational Physics of Natural
Phenomena (Cosmogrid). The original wavelet software was provided by C. Torrence and G. Compo, and is available at URL: http://paos. colorado. edu/research/wavelets/.

\section{References}

Aschwanden, M. J., De Pontieu, B. Schrijver, C. J., \& Title, A. M. 2002, Sol. Phys., 206, 99

Bogdan, T. J. 2000, Sol. Phys., 192, 373

Banerjee, D., O'Shea, E., Doyle, J. G., \& Goossens, M. 2001, A\&A, 380, L39

Banerjee, D., O'Shea, E., Goossens, M., Doyle, J. G., \& Poedts, S. 2002, A\&A, 395, 263

Berghmans, D., Clette, F. 1999, Sol. Phys., 186, 207

Brynildsen, N., Leifsen, T., Kjeldseth-Moe, O., Maltby, P., \& Wilhelm, K. 1999a, ApJ, 511, L121

Brynildsen, N., Kjeldseth-Moe, O., Maltby, P., \& Wilhelm, K. 1999b, ApJ, 517, L159

Brynildsen, N., Maltby, P., Leifsen, T., Kjeldseth-Moe, O., \& Wilhelm, K. 2000, Sol. Phys., 191, 129

Brynildsen, N., Maltby, P., Fredvik, T., \& Kjeldseth-Moe, O. 2002, Sol. Phys., 207, 259

Brynildsen, N., Maltby, P., Foley, C., Fredvik, T., \& Kjeldseth-Moe, O. 2004, Sol. Phys., 221, 237

Deforest, C. E., \& Gurman, J. B. 1998, ApJ, 501, L217

Del Zanna, G. 1999, Ph.D. dissertation

De Moortel, I., Ireland, J., \& Walsh, R. W. 2000, A\&A, 355, L23

De Moortel, I., Hood, A. W., \& Ireland, J. 2002, A\&A, 381, 311

Doyle, J. G., van den Oord, G. H. J., O’Shea, E., \& Banerjee, D. 1998, Sol. Phys., 181, 51

Doyle, J. G., Dzifcakova, E., \& Madjarska, M. S. 2003, Sol. Phys., 218,79

Fludra, A. 1999, A\&A, 344, L75

Fludra, A. 2001, A\&A, 368, 639

Harrison, R. A., Sawyer, E. C., Carter, M. K., et al. 1995, Sol. Phys., 162,233

King, D. B., Nakariakov, V. M., DeLuca, E. E., et al. 2003, A\&A, 404, L1

Klimchuk, J. A., Taner, S. E. M., \& De Moortel, I. 2004, A\&AS, 204, 9503

Maltby, P., Brynildsen, N., Fredvik, T., Kjeldseth-Moe, O., \& Wilhelm, K. 1999, Sol. Phys., 190, 437

Maltby, P., Brynildsen, N., Kjeldseth-Moe, O., \& Wilhelm, K. 2001, A\&A, 373, L1

Nakariakov, V. M., Ofman, L., Deluca, E. E., Roberts, B., \& Davila, J. M. 1999, Science, 285, 862

Nemec, A. F., \& Nemec, J. M. 1985, AJ, 90, 2317

Noyes, R. W., Raymond, J. C., Doyle, J. G., \& Kingston, A. E. 1985, ApJ, 297, 805

Ofman, L., Romoli, M., Poletto, G., Noci, G., \& Kohl, J. L. 1997, ApJ, 491, L1110

O’Shea, E., Banerjee, D., Doyle, J. G., Fleck, B., \& Murtagh, F. 2001, A\&A, 368, 1095

O’Shea, E., Muglach, K., \& Fleck, B. 2002, A\&A, 387, 642

Robbrecht, E., Verwichte, E., Berghmans, D., et al. 2001, A\&A, 370, 591

Sakurai, T., Ichimoto, K., Raju, K.P., et al. 2002, Sol. Phys., 209, 265

Schrijver, C. J., Aschwanden, M. J., \& Title, A. M. 2002, Sol. Phys., 206, 69S

Thomas, J. H., Lites, B. W., Gurman, J. B., \& Ladd, E. F. 1987, ApJ, 312,457

Torrence, C., \& Compo, G. P. 1998, Bull. Amer. Meteor. Soc., 79, 61

Tziotziou, K., Tsiropoula, G., \& Mein, P. 2002, A\&A, 381, 279

Zhukov, V. I. 2002, A\&A, 386, 653 